\title{
On a Subclass of Analytic Functions Related to a Hyperbola
}

\author{
Jagannath Patel $^{1}$ and Ashok Kumar Sahoo ${ }^{2}$ \\ ${ }^{1}$ Department of Mathematics, Utkal University, Vani Vihar, Bhubaneswar 751004, India \\ ${ }^{2}$ Department of Mathematics, Institute of Technical Education and Research, Jagmohan Nagar, Khandagiri, \\ Bhubaneswar 751030, India
}

Correspondence should be addressed to Jagannath Patel; jpatelmath@yahoo.co.in

Received 14 January 2014; Accepted 12 April 2014; Published 7 May 2014

Academic Editor: Gelu Popescu

Copyright (c) 2014 J. Patel and A. K. Sahoo. This is an open access article distributed under the Creative Commons Attribution License, which permits unrestricted use, distribution, and reproduction in any medium, provided the original work is properly cited.

The object of the present investigation is to solve Fekete-Szegö problem and determine the sharp upper bound to the second Hankel determinant for a new class $\widetilde{R}(a, c, \rho)$ of analytic functions in the unit disk. We also obtain a sufficient condition for an analytic function to be in this class.

\section{Introduction and Preliminaries}

Let $\mathscr{A}$ be the class of functions $f$ of the form:

$$
f(z)=z+\sum_{n=2}^{\infty} a_{n} z^{n}
$$

which are analytic in the open unit disk $\mathscr{U}=\{z \in \mathbb{C}:|z|<1\}$.

A function $f \in \mathscr{A}$ is said to be starlike function of order $\rho$ and convex function of order $\rho$, respectively, if and only if $\operatorname{Re}\left\{z f^{\prime}(z) / f(z)\right\}>\rho$ and $\operatorname{Re}\left\{1+\left(z f^{\prime \prime}(z) / f^{\prime}(z)\right)\right\}>\rho$, for $0 \leq \rho<1$ and for all $z \in \mathcal{U}$. By usual notations, we denote these classes of functions by $\mathcal{S}^{\star}(\rho)$ and $\mathscr{K}(\rho)(0 \leq \rho<1)$, respectively. We write $\mathcal{S}^{\star}(0)=\mathcal{S}^{\star}$ and $\mathscr{K}(0)=\mathscr{K}$, the familiar subclasses of starlike functions and convex functions in $\mathcal{U}$.

Furthermore, a function $f \in \mathscr{A}$ is said to in the class $\mathscr{R}(\rho)$, if it satisfies the inequality:

$$
\operatorname{Re}\left\{f^{\prime}(z)\right\}>\rho \quad(0 \leq \rho<1 ; z \in \mathcal{U}) .
$$

Note that $\mathscr{R}(\rho)$ is a subclass of close-to-convex functions of order $\rho(0 \leq \rho<1)$ in $\mathcal{U}$.

Let $\mathscr{P}$ denote the class of analytic functions of the form:

$$
\phi(z)=1+p_{1} z+p_{2} z^{2}+\cdots \quad(z \in \mathscr{U})
$$

satisfying the condition $\operatorname{Re}\{\phi(z)\}>0$ in $\mathcal{U}$.
Let the functions $f$ and $g$ be analytic in $\mathbb{U}$. We say that $f$ is subordinate to $g$, written as $f<g$ or $f(z) \prec g(z)(z \in \mathbb{U})$, if there exists a Schwarz function $\omega$, which (by definition) is analytic in $\mathbb{U}$ with $\omega(0)=0,|\omega(z)|<1$ and $f(z)=$ $g(\omega(z)), z \in \mathbb{U}$. Furthermore, if the function $g$ is univalent in $\mathbb{U}$, then we have the following equivalence relation (cf., e.g., [1]):

$$
f(z) \prec g(z) \Longleftrightarrow f(0)=g(0), \quad f(\mathbb{U}) \subset g(\mathbb{U}) .
$$

For the functions $f, g$ analytic in $\mathcal{U}$ and given by the power series

$$
f(z)=\sum_{n=0}^{\infty} a_{n} z^{n}, \quad g(z)=\sum_{n=0}^{\infty} b_{n} z^{n},
$$

their Hadamard product (or convolution), denoted by $f \star g$ is defined as

$$
(f \star g)(z)=\sum_{n=0}^{\infty} a_{n} b_{n} z^{n}=(g \star f)(z) \quad(z \in \mathcal{U}) .
$$

Note that $f \star g$ is analytic in $\mathcal{U}$.

The Gauss hypergeometric function ${ }_{2} F_{1}$ is defined by the infinite series

$$
{ }_{2} F_{1}(a, b ; c ; z)=\sum_{n=0}^{\infty} \frac{(a)_{n}(b)_{n}}{(c)_{n}} \frac{z^{n}}{n !}
$$

$$
\left(a, b, c \in \mathbb{C}, c \notin \mathbb{Z}_{0}^{-}=\{0,-1,-2, \ldots\} ; z \in \mathcal{U}\right),
$$


where $(\kappa)_{n}$ denotes the Pochhammer symbol (or shifted factorial) given, in terms of the Gamma function $\Gamma$, by

$$
(\kappa)_{n}=\frac{\Gamma(\kappa+n)}{\Gamma(\kappa)}= \begin{cases}\kappa(\kappa+1) \cdots(\kappa+n-1), & n \in \mathbb{N} \\ 1, & n=0 .\end{cases}
$$

We note that the series, given by (7), converges absolutely for $z \in \mathcal{U}$ and hence the function ${ }_{2} F_{1}$ represents an analytic function in the unit disc $\mathscr{U}$ [2].

We further observe that the Gauss hypergeometric function ${ }_{2} F_{1}$ plays an important role in the study of various properties and characteristics of subclasses of univalent/multivalent functions in geometric function theory (cf., e.g. [3-5]). In our present investigation, we consider the incomplete beta function $\psi$, defined by

$$
\begin{array}{r}
\psi(a, c ; z)=z_{2} F_{1}(a, 1 ; c ; z)=\sum_{n=0}^{\infty} \frac{(a)_{n}}{(c)_{n}} z^{n+1} \\
\left(a, c \in \mathbb{C}, c \notin \mathbb{Z}_{0}^{-} ; z \in \mathcal{U}\right) .
\end{array}
$$

By making use of the Hadamard product and the function $\psi$, Carlson and Shaffer [6] defined the linear operator $\mathscr{L}(a, c): \mathscr{A} \rightarrow \mathscr{A}$ by

$$
\mathscr{L}(a, c) f(z)=\psi(a, c ; z) \star f(z) \quad(f \in \mathscr{A} ; z \in \mathscr{U}) .
$$

If $f \in \mathscr{A}$ is given by (1), then it follows from (10) that

$$
\begin{aligned}
& \mathscr{L}(a, c) f(z)=z+\sum_{n=1}^{\infty} \frac{(a)_{n}}{(c)_{n}} a_{n+1} z^{n+1} \quad(z \in \mathscr{U}), \\
& z(\mathscr{L}(a, c) f)^{\prime}(z) \\
&=a \mathscr{L}(a+1, c) f(z)-(a-1) \mathscr{L}(a, c) f(z) \quad(z \in \mathscr{U}) .
\end{aligned}
$$

The operator $\mathscr{L}(a, c)$ extends several operators introduced and studied by earlier researchers in geometric function theory. For example, $\mathscr{L}(m+1,1) f(z)=\mathscr{D}^{m} f(z)(f \in$ $\mathscr{A}, m \in \mathbb{Z}, m>-1 ; z \in \mathscr{U})$, the well-known Ruscheweyh derivative operator [7] of $f$ and $\mathscr{L}(2,2-\lambda) f(z)=$ $\Omega_{z}^{\lambda} f(z)(f \in \mathscr{A}, 0 \leq \lambda<1 ; z \in \mathscr{U})$, the familiar OwaSrivastava fractional differential operator [8] of $f$.

With the aid of the linear operator $\mathscr{L}(a, c)$, we introduce a subclass of $\mathscr{A}$ as follows.

Definition 1. A function $f \in \mathscr{A}$ is said to be in the class $\widetilde{R}(a, c, \rho)$, if it satisfies the following subordination relation:

$$
\frac{\mathscr{L}(a, c) f(z)}{z} \prec\left\{\frac{1+(1-2 \rho) z}{1-z}\right\}^{1 / 2} \quad(0 \leq \rho<1 ; z \in \mathscr{U}),
$$

where the power in the right hand side of (13) indicates the principal branch. Note that if $f \in \widetilde{\mathscr{R}}(a, c, \rho)$, then by (13)

$$
\frac{\mathscr{L}(a, c) f(z)}{z}=\{\rho+(1-\rho) \phi(z)\}^{1 / 2} \quad(\phi \in \mathscr{P} ; z \in \mathscr{U}) .
$$

We denote by $\widetilde{\mathscr{R}}(2,1, \rho)=\widetilde{\mathscr{R}}(\rho)$, the class of functions $f \in \mathscr{A}$ satisfying the subordination condition:

$$
f^{\prime}(z) \prec\left\{\frac{1+(1-2 \rho) z}{1-z}\right\}^{1 / 2} \quad(0 \leq \rho<1 ; z \in \mathcal{U}) .
$$

In fact, by suitably specializing the parameters $a, c$, and $\rho$ in the class $\widetilde{R}(a, c, \rho)$, we can obtain several subclasses of $\mathscr{A}$.

Remark 2. To bring out the geometrical significance of the class $\widetilde{R}(a, c, \rho)$, we set

$$
h_{\rho}(z)=\left\{\frac{1+(1-2 \rho) z}{1-z}\right\}^{1 / 2} \quad(0 \leq \rho<1 ; z \in \mathscr{U})
$$

and note that

$$
\omega=h_{\rho}\left(e^{i \theta}\right)=\frac{1+(1-2 \rho) e^{i \theta}}{1-e^{i \theta}} \quad(0 \leq \theta \leq 2 \pi)
$$

which gives $e^{i \theta}\left(\omega^{2}-2 \rho+1\right)=\omega^{2}-1$ or $\left|\omega^{2}-1\right|=\left|\omega^{2}+1-2 \rho\right|$. Letting $\omega=u+i v$, we deduce that

$$
1-\left(u^{2}-v^{2}\right)^{2}+4 u^{2} v^{2}=\left(u^{2}-v^{2}+1-2 \rho\right)^{2}+4 u^{2} v^{2},
$$

which on simplification reduces to $u^{2}-v^{2}=\rho$. Thus, $h_{\rho}(\mathscr{U})$ is the interior of the right half branch of the hyperbola $u^{2}-$ $v^{2}=\rho$. Hence, if $f \in \widetilde{\mathscr{R}}(a, c, \rho)$, then the set of values $\mathscr{L}(a, c) f(z) / z$ for $z \in \mathcal{U}$ lie in $h_{\rho}(\mathcal{U})$, where $h_{\rho}$ is given by (16).

Fekete and Szegö [9] defined the Hankel determinant of a function $f$, given by (1) as

$$
H_{2}(1)=\left|\begin{array}{ll}
a_{1} & a_{2} \\
a_{2} & a_{3}
\end{array}\right|=a_{3}-a_{2}^{2} \quad\left(a_{1}=1\right) .
$$

In our present investigation, we also consider the second Hankel determinant of $f$, given by

$$
H_{2}(2)=\left|\begin{array}{ll}
a_{2} & a_{3} \\
a_{3} & a_{4}
\end{array}\right|=a_{2} a_{4}-a_{3}^{2} .
$$

It is known [10] that if $f$ given by (1) is analytic and univalent in $\mathcal{U}$, then the sharp inequality $H_{2}(1)=\left|a_{3}-a_{2}^{2}\right| \leq 1$ holds. For a family $\mathscr{F}$ of functions in $\mathscr{A}$ of the form (1), the more general problem of finding the sharp upper bounds for the functionals $\left(a_{3}-\mu a_{2}^{2}\right)(\mu \in \mathbb{R} / \mathbb{C})$ is popularly known as Fekete-Szegö problem for the class $\mathscr{F}$. The Fekete-Szegö problem for the known classes of univalent functions, starlike functions, convex functions, and close-to-convex functions has been completely settled $[9,11-18]$. Recently, Janteng et al. $[19,20]$ have obtained the sharp upper bounds to the second Hankel determinant $\mathrm{H}_{2}(2)$ for the family $\mathscr{R}$ of functions in $\mathscr{A}$ whose derivatives have positive real part in $\mathcal{U}$. For initial work on the class $\mathscr{R}$, one may refer to the paper by MacGregor [21].

Our objective in the present paper is to solve the FeketeSzegö problem and also to determine the sharp upper bound 
to the second Hankel determinant for the class $\widetilde{\mathscr{R}}(a, c, \rho)$ by following the techniques devised by Libera and Złotkiewicz $[22,23]$. The criteria for functions in $\mathscr{A}$ to be in this class are also obtained.

To establish our main results, we will need the following results about the functions belonging to the class $\mathscr{P}$.

Lemma 3. Let the function $\phi$, given by (3), be a member of the class $\mathscr{P}$. Then

$$
\begin{gathered}
\left|p_{k}\right| \leq 2 \quad(k \geq 1), \\
\left|p_{2}-v p_{1}^{2}\right| \leq 2 \max \{1,|2 v-1|\} \quad(\nu \in \mathbb{C}), \\
p_{2}=\frac{1}{2}\left\{p_{1}^{2}+\left(4-p_{1}^{2}\right) x\right\}, \\
p_{3}=\frac{1}{4}\left\{p_{1}^{3}+2\left(4-p_{1}^{2}\right) p_{1} x-\left(4-p_{1}^{2}\right) p_{1} x^{2}\right. \\
\left.+2\left(4-p_{1}^{2}\right)\left(1-|x|^{2}\right) z\right\}
\end{gathered}
$$

for some complex numbers $x, z$ satisfying $|x| \leq 1$ and $|z| \leq 1$. The estimates in (21) and (22) are sharp.

We note that the estimate (21) is contained in [10]; the estimate (22) is obtained by Ma and Minda [24]; the results in (23) and (24) are due to Libera and Złotkiewicz [23] (see also [22]).

\section{Main Results}

Unless otherwise mentioned, we assume throughout the sequel that

$$
a>0, \quad c>0, \quad 0 \leq \rho<1 .
$$

Now, we determine the sharp upper bound for the functional $\left|a_{3}-\mu a_{2}^{2}\right|(\mu \in \mathbb{C})$ for functions of the form (1) belonging to the class $\widetilde{\mathscr{R}}(a, c, \rho)$.

Theorem 4. Let $a>0$ and $c>0$. If the function $f$, given by (1), belongs to the class $\widetilde{\mathscr{R}}(a, c, \rho)$, then for any $\mu \in \mathbb{C}$

$$
\begin{aligned}
\mid a_{3} & -\mu a_{2}^{2} \mid \\
\leq & \frac{(c)_{2}(1-\rho)}{(a)_{2}} \\
& \times \max \left\{1, \frac{|2(a+1) c(1-\rho) \mu-a(c+1)(1+\rho)|}{2 a(c+1)}\right\} .
\end{aligned}
$$

The estimate in (26) is sharp.

Proof. Since $f \in \widetilde{\mathscr{R}}(a, c, \rho)$, by (14) we have

$$
\frac{\mathscr{L}(a, c) f(z)}{z}=\{\rho+(1-\rho) \phi(z)\}^{1 / 2} \quad(z \in \mathcal{U}),
$$

where $\phi \in \mathscr{P}$ is given by (3). It is easily seen that

$$
\begin{aligned}
&\{\rho+(1-\rho) \phi(z)\}^{1 / 2} \\
&= 1+\frac{1}{2}(1-\rho) p_{1} z+\frac{1-\rho}{2}\left\{p_{2}-\frac{(1-\rho)}{4} p_{1}^{2}\right\} z^{2} \\
&+\frac{1-\rho}{2}\left\{p_{3}-\frac{(1-\rho)}{2} p_{1} p_{2}+\frac{(1-\rho)^{2}}{8} p_{1}^{3}\right\} z^{3}+\cdots \\
&(z \in \mathcal{U}) .
\end{aligned}
$$

Writing the series expansion of $\mathscr{L}(a, c) f(z)$ given by (11), $\{\rho+(1-\rho) \phi(z)\}^{1 / 2}$, in (27) and equating the coefficients of $z, z^{2}, z^{3}$ in the resulting equation, we obtain

$$
\begin{gathered}
a_{2}=\frac{(1-\rho) c}{2 a} p_{1}, \\
a_{3}=\frac{(1-\rho)(c)_{2}}{2(a)_{2}}\left\{p_{2}-\frac{(1-\rho)}{4} p_{1}^{2}\right\}, \\
a_{4}=\frac{(1-\rho)(c)_{3}}{2(a)_{3}}\left\{p_{3}-\frac{(1-\rho)}{2} p_{1} p_{2}+\frac{(1-\rho)^{2}}{8} p_{1}^{3}\right\} .
\end{gathered}
$$

Thus for any $\mu \in \mathbb{C}$,

$$
\begin{aligned}
& \left|a_{3}-\mu a_{2}^{2}\right| \\
& \quad=\frac{(c)_{2}(1-\rho)}{2(a)_{2}}\left|p_{2}-\frac{(1-\rho)\{a(c+1)+2(a+1) c \mu\}}{4 a(c+1)} p_{1}^{2}\right|
\end{aligned}
$$

and by using (22) in the above expression, we get

$$
\begin{aligned}
& \left|a_{3}-\mu a_{2}^{2}\right| \\
& \leq \frac{(c)_{2}(1-\rho)}{(a)_{2}} \\
& \quad \times \max \left\{1,\left|\frac{(1-\rho)}{2 a(c+1)}\{a(c+1)+2(a+1) c \mu\}-1\right|\right\}
\end{aligned}
$$

which, upon simplification, gives the required assertion of Theorem 4.

Equality in (26) holds for the function $f_{0}$ defined in $\mathcal{U}$ by

$f_{0}(z)$

$$
=\left\{\begin{array}{l}
\psi(c, a ; z) \star z h_{\rho}(z), \\
\frac{|2(a+1) c(1-\rho) \mu-a(c+1)(1+\rho)|}{2 a(c+1)} \leq 1, \\
\psi(c, a ; z) \star z h_{\rho}\left(z^{2}\right), \\
\frac{|2(a+1) c(1-\rho) \mu-a(c+1)(1+\rho)|}{2 a(c+1)}>1,
\end{array}\right.
$$

where the function $h_{\rho}$ is given by (16). This completes the proof of Theorem 4 . 
Theorem 5. Let $a>0, c>0$ and $\mu \in \mathbb{R}$. If the function $f$, given by (1), belongs to the class $\widetilde{\mathscr{R}}(a, c, \rho)$, then

$$
\begin{aligned}
& \left|a_{3}-\mu a_{2}^{2}\right| \\
& \leq\left\{\begin{array}{l}
\frac{\{a(c+1)(1+\rho)-2(a+1) c(1-\rho) \mu\} c(1-\rho)}{2 a(a)_{2}} \\
\mu<-\frac{a(c+1)}{2(a+1) c} \\
\frac{(c)_{2}(1-\rho)}{(a)_{2}} \\
-\frac{a(c+1)}{2(a+1) c} \leq \mu \leq \frac{a(c+1)(3+\rho)}{2(a+1) c(1-\rho)} \\
\frac{\{2(a+1) c(1-\rho) \mu-a(c+1)(1+\rho)\} c(1-\rho)}{2 a(a)_{2}} \\
\mu>\frac{a(c+1)(3+\rho)}{2(a+1) c(1-\rho)} .
\end{array}\right.
\end{aligned}
$$

The estimates are sharp.

Proof. First, we assume that $\mu<-\{a(c+1)\} / 2(a+1) c$. Then

$$
\frac{2(a+1) c(1-\rho) \mu-a(c+1)(1+\rho)}{2 a(c+1)}<-1
$$

so that by (26), we obtain

$$
\begin{aligned}
\mid a_{3} & -\mu a_{2}^{2} \mid \\
& \leq \frac{\{a(c+1)(1+\rho)-2(a+1) c(1-\rho) \mu\} c(1-\rho)}{2 a(a)_{2}} .
\end{aligned}
$$

Next, let

$$
-\frac{a(c+1)}{2(a+1) c} \leq \mu \leq \frac{a(c+1)(3+\rho)}{2(a+1) c(1-\rho)} .
$$

Then, a routine calculation yields

$$
\frac{|2(a+1) c(1-\rho) \mu-a(c+1)(1+\rho)|}{2 a(c+1)} \leq 1
$$

and by using (26) again, we get

$$
\left|a_{3}-\mu a_{2}^{2}\right| \leq \frac{(c)_{2}(1-\rho)}{(a)_{2}} .
$$

Finally, if $\{a(c+1)(3+\rho)\} / 2(a+1) c(1-\rho)>1$, then

$$
\frac{2(a+1) c(1-\rho) \mu-a(c+1)(1+\rho)}{2 a(c+1)}>1 .
$$

Thus, by (26), we have

$$
\begin{aligned}
\mid a_{3} & -\mu a_{2}^{2} \mid \\
& \leq \frac{\{2(a+1) c(1-\rho) \mu-a(c+1)(1+\rho)\} c(1-\rho)}{2 a(a)_{2}} .
\end{aligned}
$$
by

The estimates are sharp for the function $f_{0}$ defined in $\mathscr{U}$

$$
\begin{aligned}
& f_{0}(z) \\
& =\left\{\begin{array}{c}
\psi(c, a ; z) \star z h_{\rho}(z), \\
\mu<-\frac{a(c+1)}{2(a+1) c} \text { or } \mu>\frac{a(c+1)(3+\rho)}{2(a+1) c(1-\rho)} \\
\psi(c, a ; z) \star z h_{\rho}\left(z^{2}\right), \\
-\frac{a(c+1)}{2(a+1) c} \leq \mu \leq \frac{a(c+1)(3+\rho)}{2(a+1) c(1-\rho)},
\end{array}\right.
\end{aligned}
$$

where the function $h_{\rho}$ is given by (16) and the proof of Theorem 5 is completed.

Using (21) in (29) and putting $\mu=0$ and $\mu=1$, respectively, in Theorem 5 , we get the following.

Corollary 6. Let $a \geq c>0$. If the function $f$, given by (1), belongs to the class $\widetilde{\mathscr{R}}(a, c, \rho)$, then

$$
\begin{gathered}
\left|a_{2}\right| \leq \frac{c(1-\rho)}{a}, \\
\left|a_{3}\right| \leq \frac{(c)_{2}(1-\rho)}{(a)_{2}}, \\
\left|a_{3}-a_{2}^{2}\right| \leq \frac{(c)_{2}(1-\rho)}{(a)_{2}} .
\end{gathered}
$$

The estimates in (44) and (46) are sharp for the function $f_{0}$ defined by

$$
f_{0}(z)=\psi(c, a ; z) \star z h_{\rho}(z) \quad(z \in \mathcal{U}),
$$

whereas the estimate in (45) is sharp for the function $f_{0}$ given by

$$
f_{0}(z)=\psi(c, a ; z) \star z h_{\rho}\left(z^{2}\right) \quad(z \in \mathscr{U}),
$$

where the function $h_{\rho}$ is given by (16).

Letting $a=2$ and $c=1$ in Theorem 8 , we obtain the following.

Corollary 7. If the function $f$, given by (1), belongs to the class $\widetilde{\mathscr{R}}(\rho)$, then

$$
\left|a_{3}-\mu a_{2}^{2}\right| \leq\left\{\begin{array}{l}
\frac{\{2(1+\rho)-3 \mu(1-\rho)\}(1-\rho)}{\mu<-\frac{2}{3}}, \\
\frac{(1-\rho)}{3}, \\
-\frac{2}{3} \leq \mu \leq \frac{2(3+\rho)}{3(1-\rho)} \\
\frac{\{3 \mu(1-\rho)-2(1+\rho)\}(1-\rho)}{12}, \\
\mu>\frac{2(3+\rho)}{3(1-\rho)} .
\end{array}\right.
$$


The estimates are sharp for the function $f_{0}$ defined in $\mathcal{U}$ by

$$
f_{0}(z)=\left\{\begin{array}{c}
\int_{0}^{z} \frac{d t}{1-t} \star z h_{\rho}(z), \\
\mu<-\frac{2}{3} \quad \text { or } \quad \mu>\frac{2(3+\rho)}{3(1-\rho)} \\
\int_{0}^{z} \frac{d t}{1-t} \star z h_{\rho}\left(z^{2}\right) \\
-\frac{2}{3} \leq \mu \leq \frac{2(3+\rho)}{3(1-\rho)}
\end{array}\right.
$$

where $h_{\rho}$ is given by (16).

Next, we find the sharp upper bound for the fourth coefficient of functions in the class $\widetilde{\mathscr{R}}(a, c, \rho)$.

Theorem 8. Let the function $f$, given by (1), belong to the class $\widetilde{\mathscr{R}}(a, c, \rho)$. Then

$$
\left|a_{4}\right| \leq \frac{(c)_{3}(1-\rho)}{(a)_{3}}
$$

and the estimate in (51) is sharp.

Proof. From (31), we have

$$
\left|a_{4}\right|=\frac{(c)_{3}(1-\rho)}{2(a)_{3}}\left|p_{3}-\frac{1-\rho}{2} p_{1} p_{2}+\frac{(1-\rho)^{2}}{8} p_{1}^{3}\right| .
$$

Since the functions $\phi(z)$ and $\phi\left(e^{i \theta} z\right)(\theta \in \mathbb{R})$ are in the class $\mathscr{P}$ simultaneously, we assume without loss of generality that $p_{1}>0$. For convenience of notation, we write $p_{1}=p(0 \leq$ $p \leq 2)$. Now, by using (23) and (24) in (52), we deduce that

$\left|a_{4}\right|$

$$
\begin{aligned}
=\frac{(c)_{3}(1-\rho)}{2(a)_{3}} \mid & \frac{1+\rho^{2}}{8} p^{3}+\frac{1+\rho}{4}\left(4-p^{2}\right) p x \\
& -\frac{1}{4}\left(4-p^{2}\right) p x^{2}+\frac{1}{2}\left(4-p^{2}\right)\left(1-|x|^{2}\right) z \mid
\end{aligned}
$$

for some complex numbers $x(|x| \leq 1)$ and $z(|z| \leq 1)$.

Applying the triangle inequality in the above expression followed by the replacement of $|x|$ with $y$ in the resulting equation, we obtain

$$
\begin{aligned}
&\left|a_{4}\right| \leq \frac{(c)_{3}(1-\rho)}{2(a)_{3}}\left\{\frac{1+\rho^{2}}{8} p^{3}+\frac{1+\rho}{4}\left(4-p^{2}\right) p y\right. \\
&\left.\quad+\frac{1}{4}\left(4-p^{2}\right)(p-2) y^{2}+\frac{1}{2}\left(4-p^{2}\right)\right\} \\
&=G(p, y) \quad(0 \leq p \leq 2,0 \leq y \leq 1) \quad \text { (say). }
\end{aligned}
$$

We next maximize the function $G(p, y)$ on the closed rectangle $[0,2] \times[0,1]$. Since

$$
\frac{\partial G}{\partial y}=\frac{1}{4}\left(4-p^{2}\right)\{p(1+\rho)-2(2-p) y\},
$$

we have $\partial G / \partial y<0$ for $0<p<2$ and $0<y<1$. Thus, $G(p, y)$ cannot have a maximum in the interior on the closed rectangle $[0,2] \times[0,1]$. Therefore, for fixed $p \in[0,2]$

$$
\max _{0 \leq y \leq 1} G(p, y)=G(p, 0)=F(p) \quad(\text { say })
$$

where

$$
F(p)=\frac{1+\rho^{2}}{8} p^{3}+\frac{1}{2}\left(4-p^{2}\right) \quad(0 \leq p \leq 2) .
$$

A routine calculation yields

$$
F^{\prime}(p)=\frac{3\left(1+\rho^{2}\right)}{8} p^{2}-p=0
$$

for $p=0$ or $p=8 /\left\{3\left(1+\rho^{2}\right)\right\}$. Since $F^{\prime \prime}(0)=-1<0$ and $F^{\prime \prime}\left(8 /\left\{3\left(1+\rho^{2}\right)\right\}\right)=1>0$, we conclude that the maximum of $F$ is attained at $p=0$. Thus, the upper bound of the function $G$ corresponds to $p=y=0$. Putting $p=y=0$ in (54), we get our desired estimate (51).

Equality in (51) holds for the function $f_{0}$ defined by

$$
f_{0}(z)=\psi(c, a ; z) \star z h_{\rho}\left(z^{3}\right) \quad(0 \leq \rho<1 ; z \in \mathcal{U}),
$$

where $h_{\rho}$ is given by (16).

In the following theorem, we find the sharp upper bound to the second Hankel determinant for the class $\widetilde{R}(a, c, \rho)$.

Theorem 9. Let $a \geq c>0$ and $(a+2)(c+1)-3(a-c)>0$. If the function $f$, given by (1), belongs to the class $\widetilde{\mathscr{R}}(a, c, \rho)$, then

$$
\left|a_{2} a_{4}-a_{3}^{2}\right| \leq\left\{\frac{(c)_{2}(1-\rho)}{(a)_{2}}\right\}^{2}
$$

The estimate in (60) is sharp.

Proof. From (29), (30), and (31), we deduce that

$$
\begin{aligned}
& \left|a_{2} a_{4}-a_{3}^{2}\right| \\
& =\frac{c(c)_{2}(1-\rho)^{2}}{4 a(a)_{2}} \\
& \times \mid\left(\frac{c+2}{a+2}\right) p_{1} p_{3}-\left(\frac{c+1}{a+1}\right) p_{2}^{2}-\frac{(a-c)(1-\rho)}{2(a+1)(a+2)} p_{1}^{2} p_{2} \\
& +\frac{\{(c+2)(a+1)+(a-c)\}(1-\rho)^{2}}{16(a+1)(a+2)} p_{1}^{4} \mid \text {. }
\end{aligned}
$$


As in Theorem 8, we assume without loss of generality that $p_{1}>0$ and for convenience of notation, we write $p_{1}=p(0 \leq$ $p \leq 2)$. By using (23) and (24) in (61), we get

$$
\begin{aligned}
& \left|a_{2} a_{4}-a_{3}^{2}\right| \\
& =\frac{c(c)_{2}(1-\rho)^{2}}{4 a(a)_{2}} \\
& \times \mid \frac{4(a-c) \rho+\{(c+2)(a+1)+(a-c)\}(1-\rho)^{2}}{16(a+1)(a+2)} p^{4} \\
& +\frac{(a-c)(1+\rho)}{4(a+1)(a+2)}\left(4-p^{2}\right) p^{2} x \\
& -\frac{\left\{4(a+2)(c+1)+(a-c) p^{2}\right\}}{4(a+1)(a+2)}\left(4-p^{2}\right) x^{2} \\
& +\frac{c+2}{2(a+2)}\left(4-p^{2}\right) p\left(1-|x|^{2}\right) z \mid \text {. }
\end{aligned}
$$

Now, by applying the triangle inequality in (62) and replacing $|x|$ by $y$ in the resulting equation, we get

$$
\begin{aligned}
& \mid \begin{array}{l}
\left|a_{2} a_{4}-a_{3}^{2}\right| \\
\leq \frac{c(c)_{2}(1-\rho)^{2}}{4 a(a)_{2}} \\
\quad \times\left\{\frac{4(a-c) \rho+\{(a+1)(c+2)+(a-c)\}(1-\rho)^{2}}{16(a+1)(c+2)} p^{4}\right. \\
\quad+\frac{c+2}{2(a+2)\left(4-p^{2}\right) p} \\
\quad+\frac{(a-c)(1+\rho)}{4(a+1)(a+2)}\left(4-p^{2}\right) p^{2} y \\
\quad+\frac{\left\{(a-c) p^{2}-2(a+1)(c+2) p+4(a+2)(c+1)\right\}}{4(a+1)(a+2)} \\
\left.\quad \times\left(4-p^{2}\right) y^{2}\right\} \quad(\text { say }) .
\end{array} \\
& =\mathscr{G}(p, y) \quad(0 \leq p \leq 2,0 \leq y \leq 1) \quad
\end{aligned}
$$

We next maximize the function $\mathscr{G}(p, y)$ on the closed rectangle $[0,2] \times[0,1]$. Since

$$
\begin{aligned}
\frac{\partial \mathscr{G}}{\partial y}= & \frac{(a-c)(1+\rho)}{4(a+1)(a+2)}\left(4-p^{2}\right) p^{2} \\
& +\frac{\{2(a+1)(c+2)-(2+p)(a-c)\}}{2(a+1)(a+2)} \\
& \times\left(4-p^{2}\right)(2-p) y>0
\end{aligned}
$$

for $0<p<2$ and $0<y<1$, it follows that $\mathscr{G}(p, y)$ cannot have a maximum in the interior on the closed rectangle $[0,2] \times[0,1]$. Thus, for fixed $p \in[0,2]$

$$
\max _{0 \leq y \leq 1} \mathscr{G}(p, y)=\mathscr{G}(p, 1)=\mathscr{F}(p) \quad \text { (say), }
$$

where

$$
\begin{aligned}
& \mathscr{F}(p) \\
& =\frac{c(c)_{2}(1-\rho)^{2}}{4 a(a)_{2}} \\
& \quad \times\left\{\frac{4(a-c) \rho+\{(a+1)(c+2)+(a-c)\}(1-\rho)^{2}}{16(a+1)(c+2)} p^{4}\right. \\
& \quad+\frac{c+2}{2(a+2)\left(4-p^{2}\right) p} \\
& \quad+\frac{(a-c)(1+\rho)}{4(a+1)(a+2)}\left(4-p^{2}\right) p^{2} \\
& \quad+\frac{\left\{(a-c) p^{2}-2(a+1)(c+2) p+4(a+2)(c+1)\right\}}{4(a+1)(a+2)} \\
& \left.\quad \times\left(4-p^{2}\right)\right\},
\end{aligned}
$$

$0 \leq \rho<1$. and $0 \leq p \leq 2$. Differentiating $\mathscr{F}$ with respect to $p$, we deduce that

$$
\begin{aligned}
& \mathscr{F}^{\prime}(p) \\
& =\frac{c(c)_{2}(1-\rho)^{2}}{4 a(a)_{2}} \\
& \quad \times\left[\frac{\{(a+1)(c+2)+(a-c)\}(1-\rho)^{2}-8(a-c)}{4(a+1)(a+2)} p^{3}\right. \\
& \left.\quad-\frac{2\{(a+2)(c+1)-(a-c)(2+\rho)\}}{(a+1)(a+2)} p\right]=0
\end{aligned}
$$

for $p=0$ or

$$
p^{2}=\frac{8\{(a+2)(c+1)-(a-c)(2+\rho)\}}{\{(a+1)(c+2)+(a-c)\}(1-\rho)^{2}-8(a-c)} .
$$

Since $p^{2}>4$ and

$$
\mathscr{F}^{\prime \prime}(0)=\frac{2\{(a+2)(c+1)-(a-c)(2+\rho)\}}{(a+1)(a+2)}<0
$$

by the hypothesis, we conclude that the maximum value of $\mathscr{F}$ is attained at $p=0$ so that the upper bound of the function $\mathscr{G}$ corresponds to $p=0$ and $y=1$. Thus, by letting $p=0$ and $y=1$ in (63), we get the estimate (60).

The estimate in (60) is sharp for the function $f_{0}$ given by (48). This completes the proof of Theorem 9. 
Putting $a=2$ and $c=1$ in Theorem 9, we get the following.

Corollary 10. If the function $f$, given by (1) belongs to the class $\widetilde{\mathscr{R}}(\rho)$, then

$$
\left|a_{2} a_{4}-a_{3}^{2}\right| \leq \frac{(1-\rho)^{2}}{9}
$$

and the estimate is sharp for the function $f_{0}$ defined by

$$
f_{0}(z)=\int_{0}^{z} \frac{d t}{1-t} \star z h_{\rho}\left(z^{2}\right) \quad(z \in \mathcal{U}),
$$

where the function $h_{\rho}$ is given by (16).

Theorem 11. Let $\gamma>0, a \geq 1 /(2 \gamma), c>0$ and $1 / 2 \leq \rho<1$. If $f \in \mathscr{A}$ satisfies the following inequality

$$
\operatorname{Re}\left\{\frac{\mathscr{L}(a+1, c) f(z)}{\mathscr{L}(a, c) f(z)}\right\}>\frac{(2 a \gamma+1) \rho-1}{2 a \gamma \rho} \quad(z \in \mathscr{U}),
$$

then

$$
\frac{\mathscr{L}(a, c) f(z)}{z} \prec\left\{\frac{1+(1-2 \rho) z}{1-z}\right\}^{1 / \gamma} \quad(z \in \mathscr{U}) .
$$

The result is the best possible.

Proof. We define the function $w$ by

$$
\frac{\mathscr{L}(a, c) f(z)}{z}=\left\{\frac{1+(1-2 \rho) w(z)}{1-w(z)}\right\}^{1 / \gamma} \quad(z \in \mathscr{U}) .
$$

Choosing the principal branch in the right hand side in (74), we note that $w$ is analytic in $\mathscr{U}$ with $w(0)=0$. Furthermore, logarithmically differentiating (74) and using the identity (12) in the resulting equation, we find that

$$
\begin{aligned}
\frac{\mathscr{L}(a+1, c) f(z)}{\mathscr{L}(a, c) f(z)}= & 1+\frac{1-2 \rho}{a \gamma} \frac{z w^{\prime}(z)}{1+(1-2 \rho) w(z)} \\
& +\frac{1}{a \gamma} \frac{z w^{\prime}(z)}{1-w(z)} \quad(z \in \mathscr{U}) .
\end{aligned}
$$

We claim that $|w(z)|<1$ for all $z \in \mathscr{U}$. If not, then there exists a point $z_{0} \in \mathcal{U}$ such that

$$
\max \left\{|w(z)|:|z| \leq\left|z_{0}\right|\right\}=\left|w\left(z_{0}\right)\right|=1 \quad\left(w\left(z_{0}\right) \neq 1\right),
$$

and let $w\left(z_{0}\right)=e^{i \theta}$. Now, by applying Jack's lemma [25], we have

$$
z_{0} w^{\prime}\left(z_{0}\right)=k w\left(z_{0}\right) \quad(k \geq 1)
$$

From (75) and (77), we obtain

$$
\begin{aligned}
& \operatorname{Re}\left\{\frac{\mathscr{L}(a+1, c) f(z)}{\mathscr{L}(a, c) f(z)}\right\} \\
&=1+\frac{k}{a \gamma} \operatorname{Re}\left(\frac{e^{i \theta}}{1-e^{i \theta}}\right) \\
&+\frac{(1-2 \rho) k}{a \gamma} \operatorname{Re}\left\{\frac{e^{i \theta}}{1+(1-2 \rho) e^{i \theta}}\right\} \\
&=1-\frac{c}{2 a \gamma}+\frac{(1-2 \rho) k}{a \gamma} \\
& \quad \times \frac{1-2 \rho+\cos \theta}{1+2(1-2 \rho) \cos \theta+(1-2 \rho)^{2}} \\
& \leq 1-\frac{c}{2 a \gamma}+\frac{(2 \rho-1) k}{2 a \gamma \rho} \\
& \leq \frac{(2 a \gamma+1) \rho-1}{2 a \gamma \rho}
\end{aligned}
$$

which contradicts the hypothesis (72). Thus, we conclude that $|w(z)|<1$ for all $z \in \mathscr{U}$ and (74) yields the required subordination relation (73).

To see that the result is the best possible, we consider the function $f_{0} \in \mathscr{A}$ defined by

$$
\begin{aligned}
& f_{0}(z)=\psi(c, a ; z) \star z\left\{\frac{1+(1-2 \rho) z}{1-z}\right\}^{1 / \gamma} \\
& \left(\gamma>0, a \geq \frac{1}{2 \gamma}, c>0, \frac{1}{2} \leq \rho<1 ; z \in \mathcal{U}\right)
\end{aligned}
$$

from which it follows that

$$
\frac{\mathscr{L}(a, c) f_{0}(z)}{z}=\left\{\frac{1+(1-2 \rho) z}{1-z}\right\}^{1 / \gamma} \quad(z \in \mathscr{U}) .
$$

Thus, $f_{0}$ satisfies the subordination relation (73). On differentiating the expression in (80) followed by the use of the identity (12) in the resulting equation, we deduce that

$$
\begin{aligned}
& \frac{\mathscr{L}(a+1, c) f_{0}(z)}{\mathscr{L}(a, c) f_{0}(z)} \\
& =1+\left(\frac{1-2 \rho}{a \gamma}\right) \frac{z}{1+(1-2 \rho) z}+\left(\frac{1}{a \gamma}\right) \frac{z}{1-z} \quad(z \in \mathcal{U}) .
\end{aligned}
$$

This implies that

$$
\frac{\mathscr{L}(a+1, c) f_{0}(z)}{\mathscr{L}(a, c) f_{0}(z)} \longrightarrow \frac{(2 a \gamma+1) \rho-1}{2 a \gamma \rho} \text { as } z \longrightarrow-1 .
$$

and the proof of Theorem 11 is completed.

In the special case $\gamma=2$, we get the following sufficient condition for the class $\widetilde{R}(a, c, \rho)$. 
Corollary 12. Let $a \geq 1 / 4, c>0$ and $1 / 2 \leq \rho<1$. If $f \in \mathscr{A}$ satisfies the following inequality:

$$
\begin{aligned}
& \operatorname{Re}\left\{\frac{\mathscr{L}(a+1, c) f(z)}{\mathscr{L}(a, c) f(z)}\right\} \\
& \quad>\frac{(4 a+1) \rho-1}{4 a}\left(\frac{1}{2} \leq \rho<1 ; z \in \mathcal{U}\right),
\end{aligned}
$$

then $f \in \widetilde{\mathscr{R}}(a, c, \rho)$. The result is the best possible for the function $f_{0}$ given by (47).

Letting $a=2, c=1$ and $\gamma=2$ in Theorem 11, we obtain the following.

Corollary 13. If $1 / 2 \leq \rho<1$ and $f \in \mathscr{A}$ satisfies

$$
\operatorname{Re}\left\{1+\frac{f^{\prime \prime}(z)}{f^{\prime}(z)}\right\}>\frac{5 \rho-1}{4 \rho} \quad\left(\frac{1}{2} \leq \rho<1 ; z \in \mathcal{U}\right),
$$

then $f \in \widetilde{R}(\rho)$. The result is the best possible for the function $f_{0}$ defined by

$$
f_{0}(z)=\int_{0}^{z} \frac{d t}{1-t} \star z h_{\rho}(z) \quad\left(\frac{1}{2} \leq \rho<1 ; z \in \mathcal{U}\right),
$$

where the function $h_{\rho}$ is given by (16).

Theorem 14. Let $a>0, c>0$ and $\gamma>0$. If $f \in \mathscr{A}$ satisfies the following subordination relation:

$$
\begin{aligned}
& \frac{\mathscr{L}(a, c) f(z)}{z} \\
& <\left\{\frac{1+(1-2 \rho) z}{1-z}\right\}^{1 / \gamma} \quad\left(\frac{1}{2} \leq \rho<1 ; z \in \mathcal{U}\right),
\end{aligned}
$$

then

$$
\operatorname{Re}\left(\frac{\mathscr{L}(a+1, c) f(z)}{\mathscr{L}(a, c) f(z)}\right)>\rho \quad\left(|z|<r_{0}(a, \gamma, \rho)\right),
$$

where

$$
\begin{aligned}
& r_{0}(a, \gamma, \rho) \\
& = \begin{cases}\frac{(1+a \gamma \rho)-\sqrt{(1+a \gamma \rho)^{2}-(a \gamma)^{2}(2 \rho-1)}}{a \gamma(2 \rho-1)}, & \frac{1}{2}<\rho<1 \\
\frac{a \gamma}{2+a \gamma}, & \rho=\frac{1}{2} .\end{cases}
\end{aligned}
$$

The bound $r_{0}(a, \gamma, \rho)$ in (88) is the best possible.

Proof. From (86), we get

$$
\left(\frac{\mathscr{L}(a, c) f(z)}{z}\right)=\{\rho+(1-\rho) \phi(z)\}^{1 / \gamma} \quad(\phi \in \mathscr{P} ; z \in \mathscr{U}),
$$

where we choose the principal branch in (89). Taking logarithmic differentiation in (89) and using the identity (12) in the resulting equation, we deduce that

$$
\begin{aligned}
& \operatorname{Re}\left\{\frac{\mathscr{L}(a+1, c) f(z)}{\mathscr{L}(a, c) f(z)}\right\}-\rho \\
& \quad \geq(1-\rho)\left[1-\frac{\left|z \phi^{\prime}(z)\right|}{a \gamma\{|\rho+(1-\rho) \phi(z)|\}}\right] \quad(z \in \mathcal{U}) .
\end{aligned}
$$

Using the following well-known estimates [21]

$$
\frac{\left|z \phi^{\prime}(z)\right|}{\operatorname{Re}\{\phi(z)\}} \leq \frac{2 r}{1-r^{2}}, \quad|\phi(z)| \leq \frac{1+r}{1-r} \quad(|z|=r<1)
$$

in (90), we get

$$
\begin{aligned}
& \operatorname{Re}\left\{\frac{\mathscr{L}(a+1, c) f(z)}{\mathscr{L}(a, c) f(z)}\right\}-\rho \\
& \quad \geq(1-\rho)\left[1-\frac{2 r}{\operatorname{a\gamma }\left\{\rho(1-r)^{2}+(1-\rho)\left(1-r^{2}\right)\right\}}\right] \\
& \quad \geq(1-\rho)\left[1-\frac{2 r}{a \gamma\left\{(2 \rho-1) r^{2}-2 \rho r+1\right\}}\right]
\end{aligned}
$$

which is certainly positive for $|z|<r_{0}(a, \gamma, \rho)$, where $r_{0}(a, \gamma, \rho)$ is given by $(88)$.

To show that the result is the best possible, we consider the function $f_{0}$ defined by

$$
\begin{array}{r}
f_{0}(z)=\psi(c, a ; z) \star z\left\{\rho+(1-\rho) \frac{1+z}{1-z}\right\}^{1 / \gamma} \\
\left(\frac{1}{2} \leq \rho<1,0<\gamma ; z \in \mathcal{U}\right) .
\end{array}
$$

Noting that

$$
\begin{aligned}
& \left\{\frac{\mathscr{L}(a+1, c) f_{0}(z)}{\mathscr{L}(a, c) f_{0}(z)}\right\}-\rho \\
& \quad=(1-\rho)\left[1+\frac{2 z}{\operatorname{a\gamma }\left\{\rho(1-z)^{2}+(1-\rho)\left(1-z^{2}\right)\right\}}\right]=0
\end{aligned}
$$

for $z=-r_{0}(a, \gamma, \rho)$, we conclude that the bound is the best possible. This proves Theorem 14.

Taking $\gamma=2$ in Theorem 14, we get the following.

Corollary 15. If $a>0, c>0,1 / 2 \leq \rho<1$ and $f \in$ $\widetilde{R}(a, c, \rho)$, then

$$
\operatorname{Re}\left(\frac{\mathscr{L}(a+1, c) f(z)}{\mathscr{L}(a, c) f(z)}\right)>\rho \quad(|z|<\kappa(a, \rho)),
$$


where

$$
\begin{aligned}
& \kappa(a, \rho) \\
& = \begin{cases}\frac{(1+2 a \rho)-\sqrt{4 a^{2}(1-\rho)^{2}+4 a \rho+1}}{2 a(2 \rho-1)}, & \frac{1}{2}<\rho<1 \\
\frac{a}{1+a}, & \rho=\frac{1}{2} .\end{cases}
\end{aligned}
$$

The bound $\kappa(a, \rho)$ is the best possible for the function $f_{0}$, given by (47).

Setting $a=2, c=1$ and $\gamma=1$ in Theorem 14, we get the following.

Corollary 16. If $f \in \mathscr{A}$ satisfies

$$
\operatorname{Re}\left\{f^{\prime}(z)\right\}>\rho \quad\left(\frac{1}{2} \leq \rho<1 ; z \in \mathcal{U}\right),
$$

then

$$
\operatorname{Re}\left\{1+\frac{z f^{\prime \prime}(z)}{f^{\prime}(z)}\right\}>2 \rho-1 \quad(|z|<\varkappa(\rho)),
$$

where

$$
\varkappa(\rho)= \begin{cases}\frac{(1+2 \rho)-\sqrt{4 \rho^{2}-4 \rho+5}}{2(2 \rho-1)}, & \frac{1}{2}<\rho<1 \\ \frac{1}{2}, & \rho=\frac{1}{2} .\end{cases}
$$

The bound $\varkappa(\rho)$ is the best possible for the function $f_{0}$, given in Corollary 13.

\section{Conflict of Interests}

The authors declare that there is no conflict of interests regarding the publication of this paper.

\section{Acknowledgments}

The authors would like to thank the reviewers for their constructive suggestions and comments which improved the presentation of the paper.

\section{References}

[1] S. S. Miller and P. T. Mocanu, Differential Subordinations: Theory and Applications, vol. 225 of Monographs and Textbooks in Pure and Applied Mathematics, Marcel Dekker, New York, NY, USA, 2000.

[2] E. T. Whittaker and G. N. Watson, A Course on Modern Analysis: An Introduction to the General Theory of Infinite Processes and Analytic Functions; With an Account of the Principal Transcendental Functions, Cambridge University Press, Cambridge, Mass, USA, 4th edition, 1927.

[3] P. Hästö, S. Ponnusamy, and M. Vuorinen, "Starlikeness of the Gaussian hypergeometric functions," Complex Variables and Elliptic Equations, vol. 55, no. 1-3, pp. 173-184, 2010.
[4] S. S. Miller and P. T. Mocanu, "Univalence of Gaussian and confluent hypergeometric functions," Proceedings of the American Mathematical Society, vol. 110, no. 2, pp. 333-342, 1990.

[5] S. Ponnusamy and M. Vuorinen, "Univalence and convexity properties for Gaussian hypergeometric functions," The Rocky Mountain Journal of Mathematics, vol. 31, no. 1, pp. 327-353, 2001.

[6] B. C. Carlson and D. B. Shaffer, "Starlike and prestarlike hypergeometric functions," SIAM Journal on Mathematical Analysis, vol. 15, no. 4, pp. 737-745, 1984.

[7] S. Ruscheweyh, "New criteria for univalent functions," Proceedings of the American Mathematical Society, vol. 49, pp. 109-115, 1975.

[8] S. Owa and H. M. Srivastava, "Univalent and starlike generalized hypergeometric functions," Canadian Journal of Mathematics, vol. 39, no. 5, pp. 1057-1077, 1987.

[9] M. Fekete and G. Szegö, "Eine bemerkung über ungerade schlichte funktionen," The Journal of the London Mathematical Society, vol. 8, no. 2, pp. 85-89, 1933.

[10] P. L. Duren, Univalent Functions, vol. 259 of Grundlehren der Mathematischen Wissenschaften, Springer, New York, NY, USA, 1983.

[11] B. Bhowmik, S. Ponnusamy, and K.-J. Wirths, "On the FeketeSzegö problem for concave univalent functions," Journal of Mathematical Analysis and Applications, vol. 373, no. 2, pp. 432438, 2011.

[12] J. H. Choi, Y. C. Kim, and T. Sugawa, "A general approach to the Fekete-Szegö problem," Journal of the Mathematical Society of Japan, vol. 59, no. 3, pp. 707-727, 2007.

[13] F. R. Keogh and E. P. Merkes, "A coefficient inequality for certain classes of analytic functions," Proceedings of the American Mathematical Society, vol. 20, pp. 8-12, 1969.

[14] W. Koepf, "On the Fekete-Szegö problem for close-to-convex functions. II," Archiv der Mathematik, vol. 49, no. 5, pp. 420433, 1987.

[15] W. Koepf, "On the Fekete-Szegö problem for close-to-convex functions," Proceedings of the American Mathematical Society, vol. 101, no. 1, pp. 89-95, 1987.

[16] R. R. London, "Fekete-Szegö inequalities for close-to-convex functions," Proceedings of the American Mathematical Society, vol. 117, no. 4, pp. 947-950, 1993.

[17] A. Pfluger, “The Fekete-Szegö inequality by a variational method," Annales Academiae Scientiarum Fennicae. Series A I. Mathematica, vol. 10, pp. 447-454, 1985.

[18] A. Pfluger, "The Fekete-Szegö inequality for complex parameters," Complex Variables. Theory and Application, vol. 7, no. 1-3, pp. 149-160, 1986.

[19] A. Janteng, S. A. Halim, and M. Darus, "Coefficient inequality for a function whose derivative has a positive real part," Journal of Inequalities in Pure and Applied Mathematics, vol. 7, no. 2, article 50, pp. 1-5, 2006.

[20] A. Janteng, S. A. Halim, and M. Darus, "Estimate on the second Hankel functional for functions whose derivative has a positive real part," The Journal of Quality Measurement and Analysis, vol. 4, pp. 189-195, 2008.

[21] T. H. MacGregor, "Functions whose derivative has a positive real part," Transactions of the American Mathematical Society, vol. 104, no. 3, pp. 532-537, 1962.

[22] R. J. Libera and E. J. Złotkiewicz, "Early coefficients of the inverse of a regular convex function," Proceedings of the American Mathematical Society, vol. 85, no. 2, pp. 225-230, 1982. 
[23] R. J. Libera and E. J. Złotkiewicz, "Coefficient bounds for the inverse of a function with derivative in $\mathscr{P}$," Proceedings of the American Mathematical Society, vol. 87, no. 2, pp. 251-257, 1983.

[24] W. C. Ma and D. Minda, "A unified treatment of some special classes of univalent functions," in Proceedings of the Conference on Complex Analysis (Tianjin, 1992), Z. Li, F. Ren, L. Yang, and S. Zhang, Eds., pp. 157-169, International Press, Cambridge, Mass, USA, 1994.

[25] I. S. Jack, "Functions starlike and convex of order $\alpha$," Journal of the London Mathematical Society, vol. 3, pp. 469-474, 1971. 


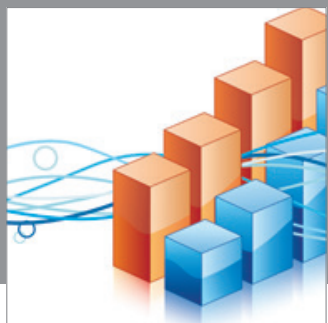

Advances in

Operations Research

mansans

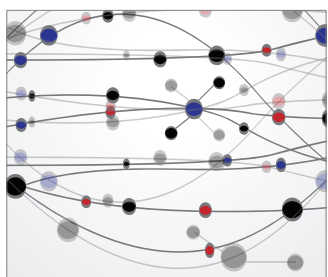

The Scientific World Journal
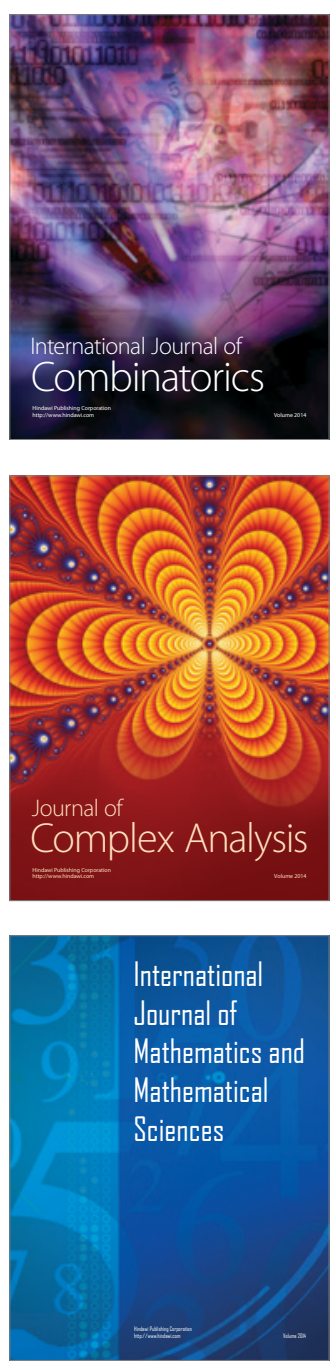
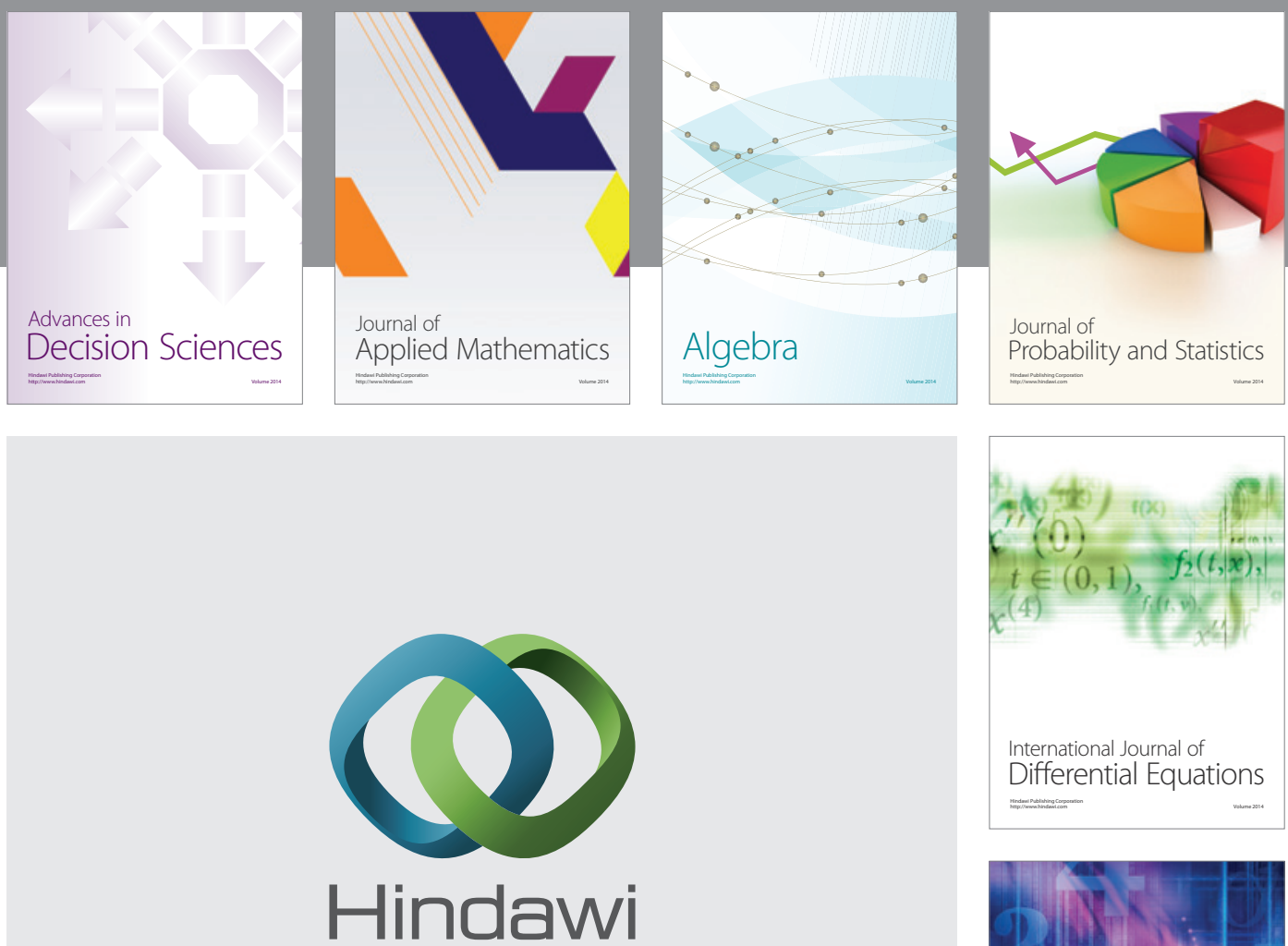

Submit your manuscripts at http://www.hindawi.com
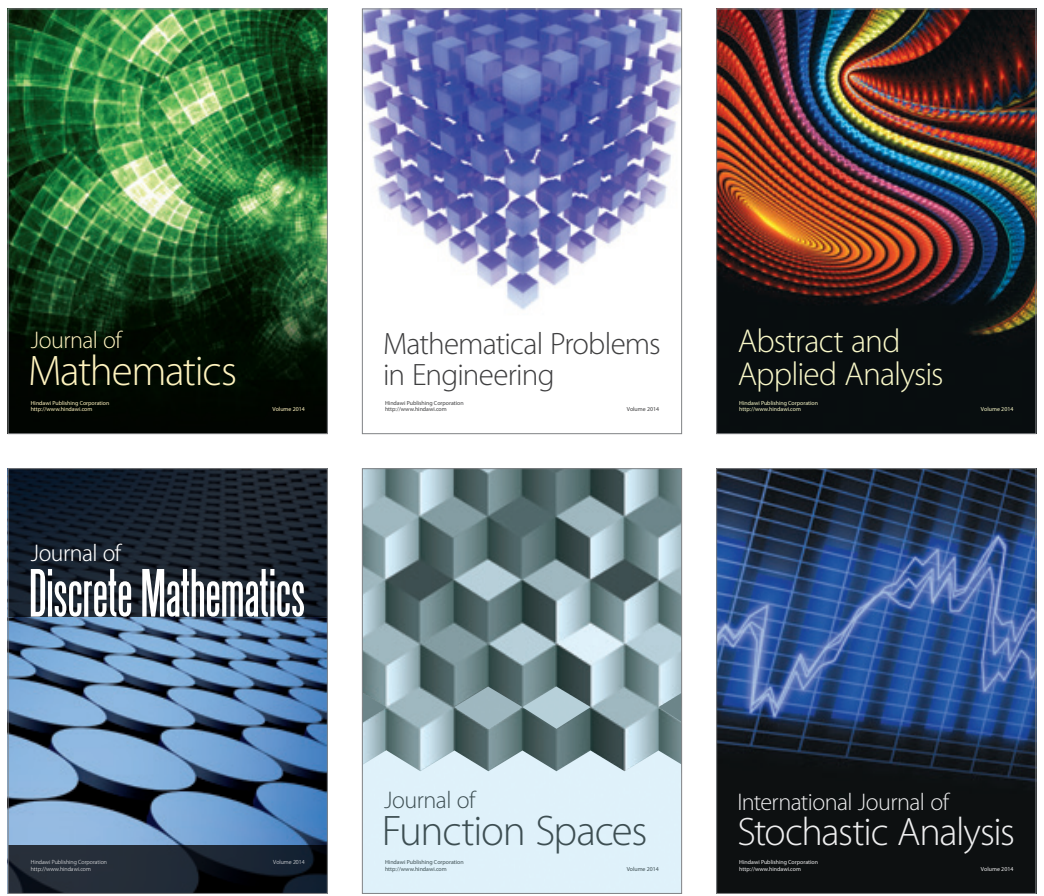

Journal of

Function Spaces

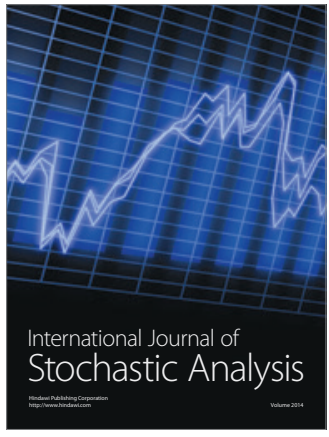

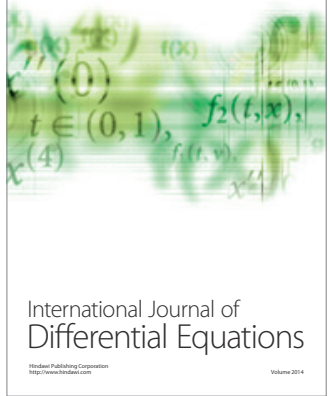
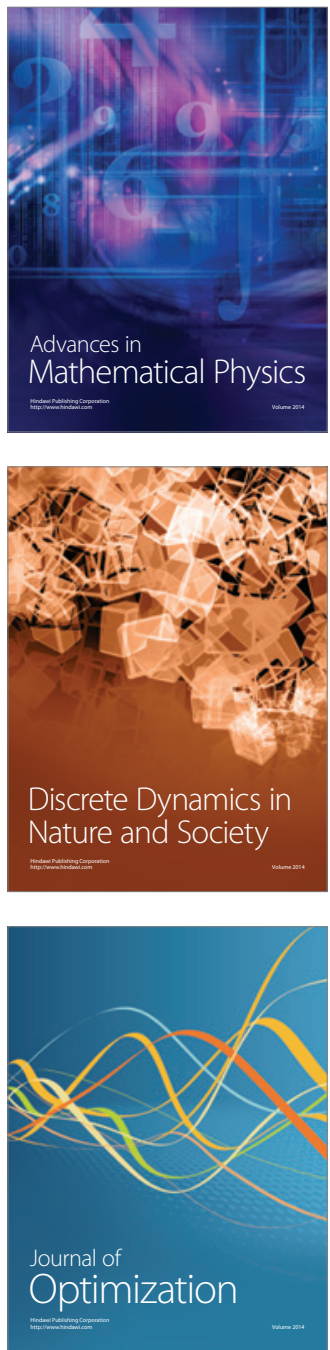